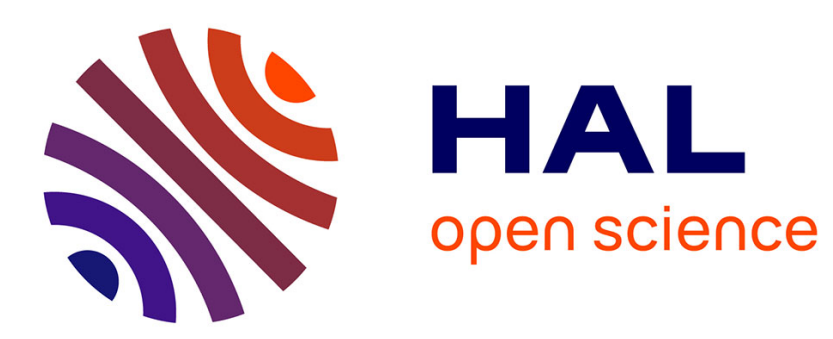

\title{
Verifying a medical protocol with temporal graphs: The case of a nosocomial disease
}

Bernard Kamsu-Foguem, Germaine Tchuenté Foguem, Clovis Foguem

\section{To cite this version:}

Bernard Kamsu-Foguem, Germaine Tchuenté Foguem, Clovis Foguem. Verifying a medical protocol with temporal graphs: The case of a nosocomial disease. Journal of Critical Care, 2014, vol. 29, pp. 690 e1-690 e9. 10.1016/j.jcrc.2014.02.006 . hal-01070512

\section{HAL Id: hal-01070512 \\ https://hal.science/hal-01070512}

Submitted on 8 Oct 2014

HAL is a multi-disciplinary open access archive for the deposit and dissemination of scientific research documents, whether they are published or not. The documents may come from teaching and research institutions in France or abroad, or from public or private research centers.
L'archive ouverte pluridisciplinaire HAL, est destinée au dépôt et à la diffusion de documents scientifiques de niveau recherche, publiés ou non, émanant des établissements d'enseignement et de recherche français ou étrangers, des laboratoires publics ou privés. 


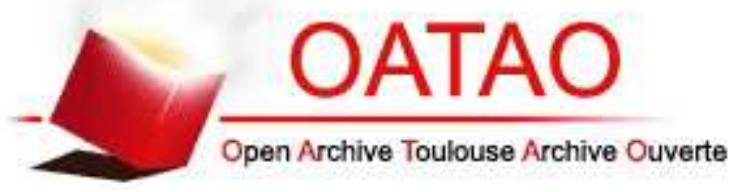

\section{Open Archive Toulouse Archive Ouverte (OATAO)}

OATAO is an open access repository that collects the work of Toulouse researchers and makes it freely available over the web where possible.

This is an author-deposited version published in: http://oatao.univ-toulouse.fr/ Eprints ID: 11814

To link to this article: DOI:10.1016/j.jcrc.2014.02.006

http://dx.doi.org/10.1016/j.jcrc.2014.02.006

\section{To cite this version:}

Kamsu Foguem, Bernard and Foguem, Clovis and Tchuenté Foguem, Germaine Verifying a medical protocol with temporal graphs: The case of a nosocomial disease. (2014) Journal of Critical Care, vol. 29. pp. 690 e1690 e9. ISSN 0883-9441 
Electronic Articles

\title{
Verifying a medical protocol with temporal graphs: The case of a nosocomial disease
}

\author{
Bernard Kamsu-Foguem, PhD, HDR ${ }^{\mathrm{a}, *}$, Germaine Tchuenté-Foguem, MSc ${ }^{\mathrm{b}}$, Clovis Foguem, MD, MSc ${ }^{\mathrm{c}}$ \\ a Laboratory of Production Engineering (LGP), EA 1905, ENIT-INPT University of Toulouse, 47 Avenue d'Azereix, BP 1629, 65016, Tarbes Cedex, France \\ ${ }^{\mathrm{b}}$ MAT Laboratory, UMI 209, Unit for Mathematical and Computer Modeling of Complex Systems - UMMISCO, Faculty of Science, University of Yaoundé I, Yaoundé, Cameroon \\ c Center for Food and Taste sciences (CSGA)-UMR 6265 CNRS-UMR 1324 INRA-University of Burgundy, Dijon, France
}

A R T I C L E I N F O

Keywords:

Visual information

Conceptual graphs

Model checking

Medical protocol

Nosocomial diseases

\begin{abstract}
A B S T R A C T
Objective: Our contribution focuses on the implementation of a formal verification approach for medical protocols with graphical temporal reasoning paths to facilitate the understanding of verification steps. Materials and methods: Formal medical guideline specifications and background knowledge are represented through conceptual graphs, and reasoning is based on graph homomorphism. These materials explain the underlying principles or rationale that guide the functioning of verifications.

Results: An illustration of this proposal is made using a medical protocol defining guidelines for the monitoring and prevention of nosocomial infections. Such infections, which are acquired in the hospital, increase morbidity and mortality and add noticeably to economic burden. An evaluation of the use of the graphical verification found that this method aids in the improvement of both clinical knowledge and the quality of actions made.

Discussion: As conceptual graphs, representations based on diagrams can be translated into computational tree logic. However, diagrams are much more natural and explicitly human, emphasizing a theoretical and practical consistency.

Conclusion: The proposed approach allows for the visual modeling of temporal reasoning and a formalization of knowledge that can assist in the diagnosis and treatment of nosocomial infections and some clinical problems. This is the first time that one emphasizes the temporal situation modeling in conceptual graphs. It will also deliver a formal verification method for clinical guideline analyses.
\end{abstract}

\section{Introduction}

A medical protocol (also known as a clinical protocol, medical guideline, or clinical guideline) is a document attesting to a good practice of a medical or paramedical action (treatment or diagnosis) according to a bibliography, a shared clinical experience, or recommendations of a consensus among professionals.

Protocol development receives attention in the medical community to the extent that these tools can improve the quality of care and reduce health care costs. Medical protocols are usually in the form of a corpus of text describing the diagnostic steps and processes of care, written in natural language. Properties that have been used to verify (model check) protocols are derived from lists of indicators of good medical practice, the latter being systematically developed by medical experts.

Nosocomial infection is a recognized public health problem worldwide with a prevalence rate of $3.0 \%$ to $20.7 \%$ and an incidence rate of $5 \%$ to $10 \%$ [1]. In Europe, the Scandinavian countries and the Netherlands, with appropriate policy measures by developing a protocol known as Search and Destroy, have a low prevalence rate of nosocomial infections

\footnotetext{
* Corresponding author. Tel.: + 336243023 37; fax: + 33562442708. E-mail address: Bernard.Kamsu-Foguem@enit.fr (B. Kamsu-Foguem).
}

$(<1 \%)$. Meanwhile, there are high prevalence rates $(>10 \%)$ in France, the United Kingdom, Germany, Belgium, and Spain. Because of this fact, it is necessary to develop a way to fight against these nosocomial infections.

Here, we work on protocols defining guidelines for the monitoring and prevention of nosocomial infections. Nosocomial infections are those infections acquired in a hospital or other healthcare facility by a patient and first appear 48 hours or more after hospital admission or within 30 days after discharge following in-patient care. In addition, occupational infections also occur among facility staff [2]. These infections are unrelated to the original illnesses that bring patients to the hospital and neither present nor incubating at the time of admission [3]. Our contribution is the visual approach, and we illustrate this with the use case of nosocomial infection.

To develop a nosocomial infection, 4 elements must be present at the level of the disease:

- An infectious agent: A variety of microorganisms are likely to lead to nosocomial infections: bacteria, viruses, fungi, and parasites. Infections may be caused by a microorganism acquired from another person in the hospital (a cross infection) or may be caused by the patient's own flora (an endogenous infection). Some organisms may be acquired from an inanimate object or substances recently contaminated from another human source (an 
environmental infection). In some regions with poor basic hygienic practices, some nosocomial infections are due to pathogens of an external origin (foodborne and airborne diseases, gas gangrene, tetanus) or were caused by microorganisms not present in the normal flora of the patients (e.g., diphtheria, tuberculosis).

- A susceptible subject: Important patient factors influencing the acquisition of an infectious agent include age, immune status, underlying disease, and diagnosis and therapeutic interventions (e.g., chemotherapy). Hospitalized patients with impaired immune systems due to chronic diseases or cancers (e.g., diabetes mellitus, respirator insufficiency, renal failure, immune disorders, burns, malignant tumors, leukemia, or acquired immunodeficiency syndrome (AIDS)) or because of their general condition (malnourished or at extreme ages of life (infancy and old age)) have an increased susceptibility to infections with opportunistic pathogens. The latter are infections with organism (s) that are normally innocuous, e.g., part of the normal human bacterial flora, but may become pathogenic when the body's immunological defenses are compromised. Immunosuppressive drugs or irradiation may lower resistance to infection. Injuries to skin or mucous membranes bypass natural defense mechanisms. Many modern diagnostic and therapeutic procedures, such as biopsies, endoscopic examinations, catheterization, intubation/ ventilation, suction and surgical procedures, increase the risk of infection. Contaminated objects or substances may be introduced directly into tissues or normally sterile sites such as the urinary tract and the lower respiratory tract.

- Environmental factors: Patients with infections or carriers of pathogenic microorganisms admitted to hospitals are potential sources of infection for patients and staff. Crowded conditions within the hospital, frequent transfers of patients from one unit to another, and concentrations of patients highly susceptible to infection in one area (eg, newborn infants, burn patients, and intensive care) all contribute to the development of nosocomial infections. Microbial flora may contaminate objects, devices, and materials, which subsequently contact susceptible body sites of patients. In addition, new infections associated with bacteria such as waterborne bacteria (atypical mycobacteria) and/or viruses and parasites continue to be identified. World Health Organization studies and others have also shown that the highest prevalence of nosocomial infections occurs in intensive care units and in acute surgical and orthopedic wards [4].

- Bacterial resistance: Many patients receive antimicrobial drugs. Through selection and the exchange of genetic resistance elements, antibiotics promote the emergence of multidrugresistant strains of bacteria and microorganisms in the normal human flora that are sensitive to the given drug and are suppressed while resistant strains persist and may become endemic in the hospital. The widespread use of antimicrobials for therapy or prophylaxis (including topical) is the major determinant of resistance. Antimicrobial agents are, in some cases, becoming less effective because of resistance. Many strains of pneumococci, staphylococci, enterococci, and tuberculosis are currently resistant to most or all antimicrobials that were once effective. Multidrug-resistant Klebsiella and Pseudomonas aeruginosa are prevalent in many hospitals. This problem is particularly critical in developing countries where more expensive second-line antibiotics may not be available or affordable.

We first present the setting of our context to provide some background. We will then review requirement specifications and finish with the verification of medical protocols. The approach presented in this article is inspired by a part of a larger research project aimed at improving the daily application of medical protocols, including a particular illustration of a medical protocol to reduce rates of nosocomial infections. The verification of a protocol can improve the quality of care and also patient safety. The protocol is the model, and the specifications are the properties. The model has to satisfy those properties.

\section{Background and context setting}

\subsection{Monitoring of nosocomial catheter-related infections}

Here, we review protocols to establish guidelines for the monitoring and prevention of nosocomial diseases that are transmitted by medical devices, such as urinary catheters, intubation probes, or catheters. The work performed in this article is based on a former study from a Spanish hospital that illustrates the application of a protocol for the management and prevention of intravenous catheter-related infections (CRIs) [5]. It was found that these infections are the most common cause of those nosocomial infections acquired in the hospital that affect morbidity and mortality and that also have economic implications (a prolonged duration of hospitalization, increased cost of health care, the emergence of multiple antibiotic resistance microorganisms, and reducing the incidence of treatment for other infections).

Taking care of and preventing nosocomial infections are the responsibility of all individuals and services providing health care (managers; physicians; and other health care workers, such as clinical microbiology, pharmacy, central supply, maintainers, housekeeping, and training services). It is recommended that transparent and appropriate evaluation criteria be used to evaluate the surveillance of nosocomial infections such as the catheter-related infections (CRI) or infections related to intravenous catheters (IRC) (Table 1). In some establishments, there are specialists in infection control, epidemiology, and infectious disease, including infection control physicians and infection control practitioners [6]. These individuals have a scientific and technical support role, for example, surveillance and research, developing and assessing policies and practical supervision, evaluating materials and products, controlling sterilization and disinfection procedures, and implementing training programs. These individuals should also support and participate in research and assessment programs at the national and international levels. A nosocomial infection prevention manual, compiling recommended instructions and practices for patient care, is an important tool [4]. The manual should be developed and updated by the infection control team with review and approval by a committee.

\subsection{Model checking}

The primary goal of model checking [7] is to check the conformity of the model with the requirements defined in the specification, with an important issue being: does the model provide an interpretation as to whether it meets or does not meet the system's requirements?

- The principle is to compare a model with a property of the system and to automatically check whether the model satisfies the property.

- Its issues: why verify the status of a model and check its outcome? There is a need to provide regulatory amendments that allow for the establishment of a new approach to safety oversight and for the certification of heath care operations. Indeed, for complex medical organizations and systems, it is necessary to have sufficient confidence in their functioning at all times.

Table 1

Simplified criteria for the surveillance of nosocomial catheter-related infections (CRI)

\begin{tabular}{ll}
\hline $\begin{array}{l}\text { Vascular catheter } \\
\text { infection }\end{array}$ & $\begin{array}{l}\text { Inflammation, lymphangitis, or purulent discharge at } \\
\text { the insertion site of the catheter, abscess, or spreading } \\
\text { cellulitis at the insertion site of the catheter during } \\
\text { the days or weeks after the intervention }\end{array}$ \\
Septicemia & $\begin{array}{l}\text { Fever or rigors, and at least rigors, and at least 1 positive } \\
\text { blood culture }\end{array}$ \\
\hline
\end{tabular}




\subsection{Requirements for medical protocols}

In this article, 4 origins of the properties of specification have been identified to meet the requirements for medical protocols. The categories of the sources are nice, and seem useful in clarifying the required properties: good medical practice, hospital characteristics, purpose of the protocol, and clinical condition of the patient. Indeed, the categories of sources play a role in the temporal graph-based verification of clinical protocols: computable inspections of a clinical protocol model or the feedback to the user if the model does not satisfy the property. The question then remains: what are they and how should they be addressed? What are medical barriers and how do they affect access to services? We are going to review what exact characteristics are associated with the main independent sources of properties in Table 2.

\subsection{Conceptual graph formalism}

The design of conceptual graphs is based on the study of perception in psychology. The meaning of a concept is reduced to its position relative to other concepts. Therefore, there is a sense with respect to a semantic net modeling of the general knowledge of the system [8]. Conceptual graphs are defined with typed objects, nodes, and edges of typed relations according to the vocabulary of the considered domain that details the allowed concepts and relationship types. The reasoning on these graphs is made via a subgraph matching mechanism by performing variable substitutions (called projections) for those making similar graphs in certain contexts. We use the structural features of graphs and semantic properties of domain vocabulary to demonstrate some equivalence classes on groups of sets [9]. These equivalence classes are used to locate information about specific knowledge-intensive activities and to help provide the scientific basis to make problem-solving approaches more consistent, guileless, and creative. Conceptual graph formalism represents a good compromise between graphic language and formal language because it facilitates the reading and understanding of the reasoning.
Conceptual graphs are a knowledge representation language, proposed by John Sowa, and allow for both rigorously defining a vocabulary (ie, ontology) and using this vocabulary to describe and reason about situations [10]. From the point-of-view of knowledge representation, conceptual graphs provide a structure of different types of knowledge that is not explicit in other formalisms, such as semantic networks or object modeling. Indeed, this formalism marks a clear separation between ontological knowledge, which is encoded in a special structure called support, and factual knowledge, which is represented by graphs. In addition, the formalism clearly distinguishes entities (represented in the graph by rectangles) and the relationships between these entities (represented by ovals).

Based on the adage that "a picture is worth a thousand words," conceptual graph operations is a superior vector for the vulgarization of the reasoning in all its forms. Without excluding demonstrations using logical formulas, graph operations are sufficient to explain the reasoning by which a pattern is often held as a demonstration until a better control of logical tools is available [11]. In a number of cases, a sequence of suitable graphical illustrations is sufficient in itself to lead to a solution. The intuition is requested more than theoretical knowledge and sometimes fragmented and inefficient.

In addition, conceptual graphs can easily be translated into the language of some other approach used in knowledge engineering, such as description logics (DL) [12], Resource Description Framework Schema [13], and its evolution OWL (Web Ontology Language) DL [14-16]. As a result, the possibility to interact and exchange knowledge modeled with internal and external staff is available to different stakeholders in the modeling system under this consideration. In addition to the visual reasoning facilities, syntactic opportunities for graphs include the intuitive ideas of graphical arguments that have no equivalent in DL [17].

However, the expressiveness of conceptual graphs may not be satisfactory for modeling the temporal knowledge of a given domain. To overcome this aforementioned drawback, we propose a formal modeling of temporal knowledge, which introduces the semantic interpretation of the temporal logic expressions of Computational Tree Logic (CTL) [18] in models of conceptual graphs. The modeling of temporal knowledge is represented by the nested conceptual

Table 2

Sources of the specification of properties

\begin{tabular}{|c|c|}
\hline Sources & Characteristic \\
\hline Good medical practice & $\begin{array}{l}\text { Concerns the aspects or factors intrinsic to the guideline by itself, such as the conditions and clinical problems it covers or the desired outcomes. } \\
\text { For example, verifying whether the guideline conforms to a good medical practice through specific requirements, such as: } \\
\text { (i) Avoidance of redundancies and inconsistencies in drug prescriptions. } \\
\text { (ii) Between } 2 \text { alternative treatments, the preferred one should always minimize side effects and allow for faster healing. } \\
\text { (iii) Paying attention to appropriate practices of hygiene (eg, hand washing, isolation, using antiseptics and disinfectants). } \\
\text { (iv) Providing direct patient care using practices that minimize infection. } \\
\text { (v) Participation in quality control techniques used to sterilize equipment in the hospital, including the selection of sterilization equipment } \\
\text { (type of appliances) and monitoring. } \\
\text { (vi) Ensuring the education and training of all staff through the support of programs on the prevention of infection using disinfection and } \\
\text { sterilization techniques. }\end{array}$ \\
\hline $\begin{array}{l}\text { Particularities of the } \\
\text { hospital }\end{array}$ & $\begin{array}{l}\text { Concerns the contextualization of the guidelines of a given hospital. The hospital care program may provide constraints and rules, taking into } \\
\text { account facts deemed to be specific knowledge and that are a reflection of local health care services. For example, the verification of a reduction } \\
\text { in the use of certain pharmacological treatments, avoiding crowded patient wards (where poor control practices may facilitate infection } \\
\text { transmission), being careful not to concentrate patients highly susceptible to infection in one area (eg, newborn infants, burn patients, intensive } \\
\text { care) or frequently transferring patients from one unit to another or the availability of specific hospital resources (such as laboratory instruments). } \\
\text { At a hospital, microbial flora may contaminate objects, devices, and materials, which subsequently contact susceptible body sites of patients. In } \\
\text { addition, new infections associated with bacteria, such as waterborne bacteria (atypical mycobacteria) and/or viruses and parasites, continue } \\
\text { to be identified. }\end{array}$ \\
\hline Guideline goal & $\begin{array}{l}\text { Concerns aspects or factors intrinsic to the guideline by itself, such as conditions and the clinical problems that it covers or the desired outcomes. } \\
\text { For example, the verification of whether the guideline contains a path requiring the specific support services required for a given treatment. }\end{array}$ \\
\hline $\begin{array}{l}\text { Patient-specific clinical } \\
\text { conditions }\end{array}$ & $\begin{array}{l}\text { Concerns the possible paths the guideline proposes to be performed given a specific patient clinical state. This state may be influenced by impaired } \\
\text { immunity, age, medical history, medical procedures, and/or invasive techniques, such as intravenous catheters, which create potential routes of } \\
\text { infection, the transmission of drug-resistant bacteria, and the severity or frequency of the nosocomial infection. For example, the verification of the } \\
\text { feasibility of a given action, the path of actions on the patient, or to prove whether there is a therapy for a patient under these clinical conditions. }\end{array}$ \\
\hline
\end{tabular}


graphs used to describe the property specification models that formalize knowledge modeling in the application domain.

Therefore, we first translated the specifications of the medical domain into formal properties. These will then be used in the reasoning process to rigorously verify that the protocol meets the requirements. We have been striving to integrate the consideration of the modeling elements of CTL into the conceptual graph modeling framework itself.

\section{Verification of medical protocols with temporal conceptual graphs}

We present a mechanism for the verification of medical protocols using conceptual graphs.

\subsection{Notion of temporal modality}

3.1.1. How do you represent temporal modalities in conceptual graphs?

The use of the conceptual graphs defined by Sowa provides a great power of representation, and the advantages of this formalism are mainly:

- A logical interpretation for representing the semantics of natural language

- A reasoning service equipped with a rigorous graph operation.

However, some of the shortcomings of this model should not be ignored: the treatment of the negation is not satisfactory and the interpretation of the modal terms is beyond the formal framework. Thus, writing sentences such as the following (complex by structure and the temporal references described) should be avoided in such protocols: "If the number of critical clinical parameters of time exceed a threshold SE, then an alarm should be provided for medical staff with remote broadcast." Therefore, our work will allow for the adequate modeling of temporal aspects for the adaptation formalization process of properties' specifications.

In his work on conceptual graphs, Bernard Moulin focused on the need for better expressive temporal representations and proposed constructions that were used to represent various types of temporal contexts: the point-of-view of the narrator, the perspective of the agent, and the temporal localization [19].
In conceptual graphs, we have the nesting graphs (embedded or a nesting of contexts) that we will use to describe the temporal modalities.

In addition, we have the following temporal terms:

\begin{tabular}{|c|c|}
\hline All: for all paths & Exists: there is a path \\
\hline$\hookrightarrow$ Finally: one day & $\hookrightarrow$ Finally: one day \\
\hline$\hookrightarrow$ Next: afterwards & $\hookrightarrow$ Next: afterwards \\
\hline$\hookrightarrow$ Until: up to & $\downarrow$ Until: up to \\
\hline L Globally: generally & \ Globally: generally \\
\hline
\end{tabular}

We note that concepts are represented by rectangles and relationships by ovals in the visual representations. Significantly, a framework based on taxonomies allows experts to represent and share their knowledge with other experts through shared and controlled vocabularies; it follows that information modeling can be improved by means of social and semantic web tools [20].

\subsection{Formalization of the three general principles of medical treatment}

We absolutely make illustrative descriptions of the 3 properties that we believe are in the users' interests to introduce the conceptual graph formalization on the origins of requirement specifications.

We translate the 3 following properties:

- First property (Fig. 1). "If a treatment T1 is not applicable to a disease in a particular medical context, then it will be replaced by an applicable treatment $\mathrm{T} 2$ that produces a positive impact on a disease similar to treatment T1."

- Second property (Fig. 2). "If a treatment T1 is not effective against a disease due to the characteristics of the disease in question, then it will be replaced by treatment $\mathrm{T} 2$, which has positive similar consequences on the disease."

- Third property (Fig. 3). "If a treatment T1 has too many negative consequences on a patient, then it will be replaced by a treatment T2 similar to T1 but having fewer negative consequences."

\subsection{Graphical modeling of a case study}

The first step is to construct domain taxonomy (hierarchy of concepts and relationships) in the target application (Tables 3 and 4).

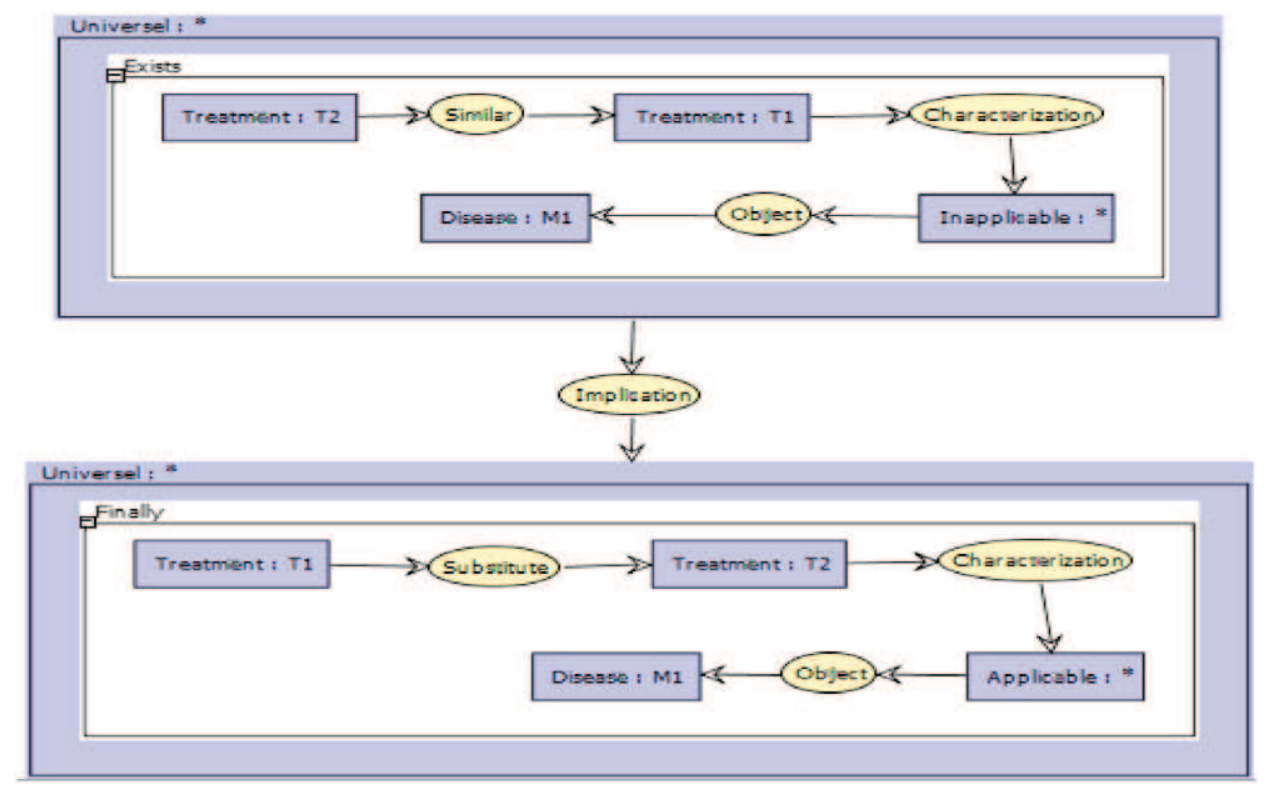

Fig. 1. First property. 


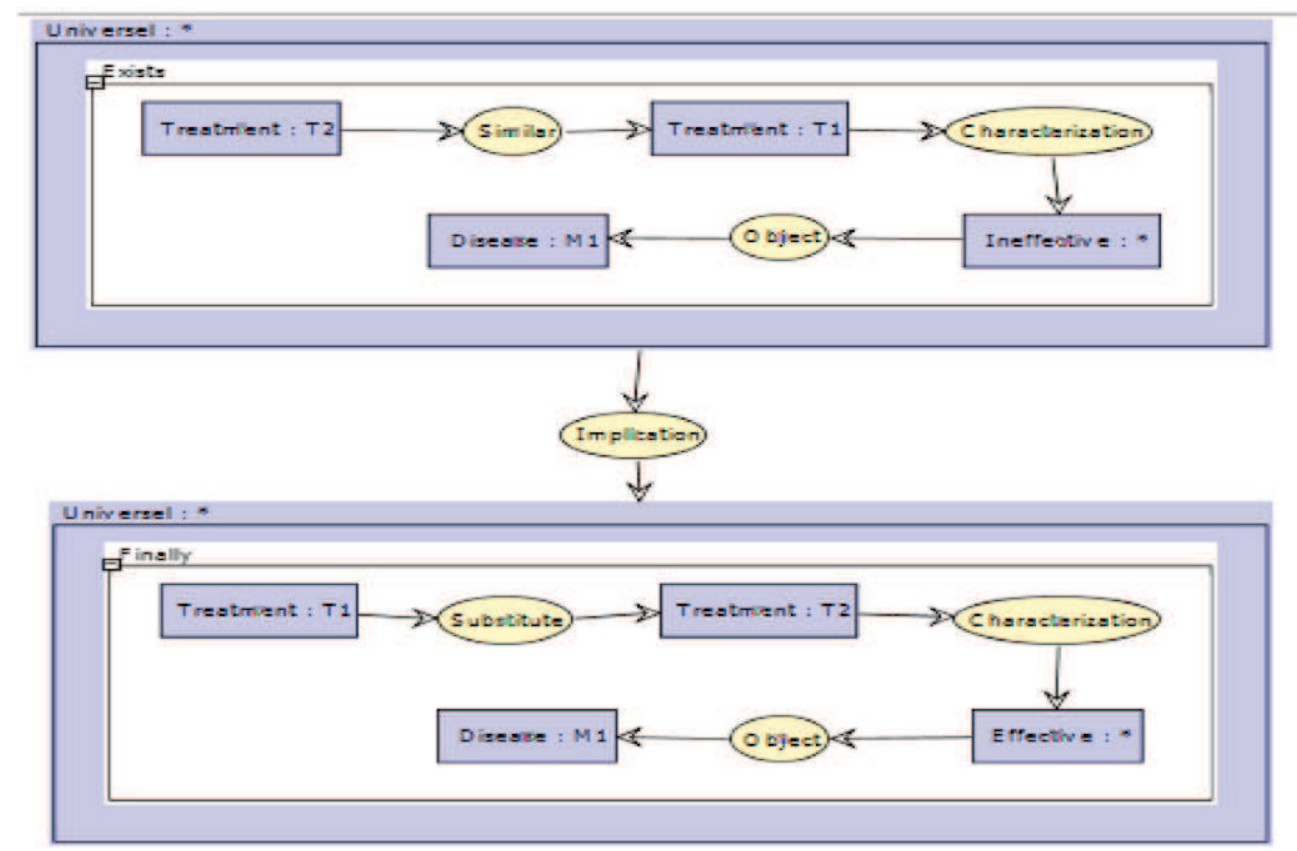

Fig. 2. Second property.

Highlighting conceptual dependencies, this taxonomy assists the process of purposeful structuring of information, but it also provides the vocabulary to be used during the model specification and verification phases with graph operations [9]. In general, the knowledge modeling encompasses the identification of a domain ontology and tasks that the system must achieve and the suitable determination of problem-solving methods to accomplish those tasks, with the definition of mappings between the domain ontology and the problem-solving methods, and the creation of application-specific content knowledge as an instantiation of the classes in the domain ontology [21].

\subsection{Verification of the catheter-related infections protocol model}

To provide a better understanding of the approach presented in this article, a particular case study is detailed using the management of catheterrelated infections (CRI) or infections related to intravenous catheters (IRC).

Infections related to intravenous catheter tests are performed for nosocomial infections contracted through a catheter and are conducted to provide evidence of a possible infection. This test has been used in a Spanish hospital and was developed based on a directive issued by the Agency for Health Care Research and Quality.

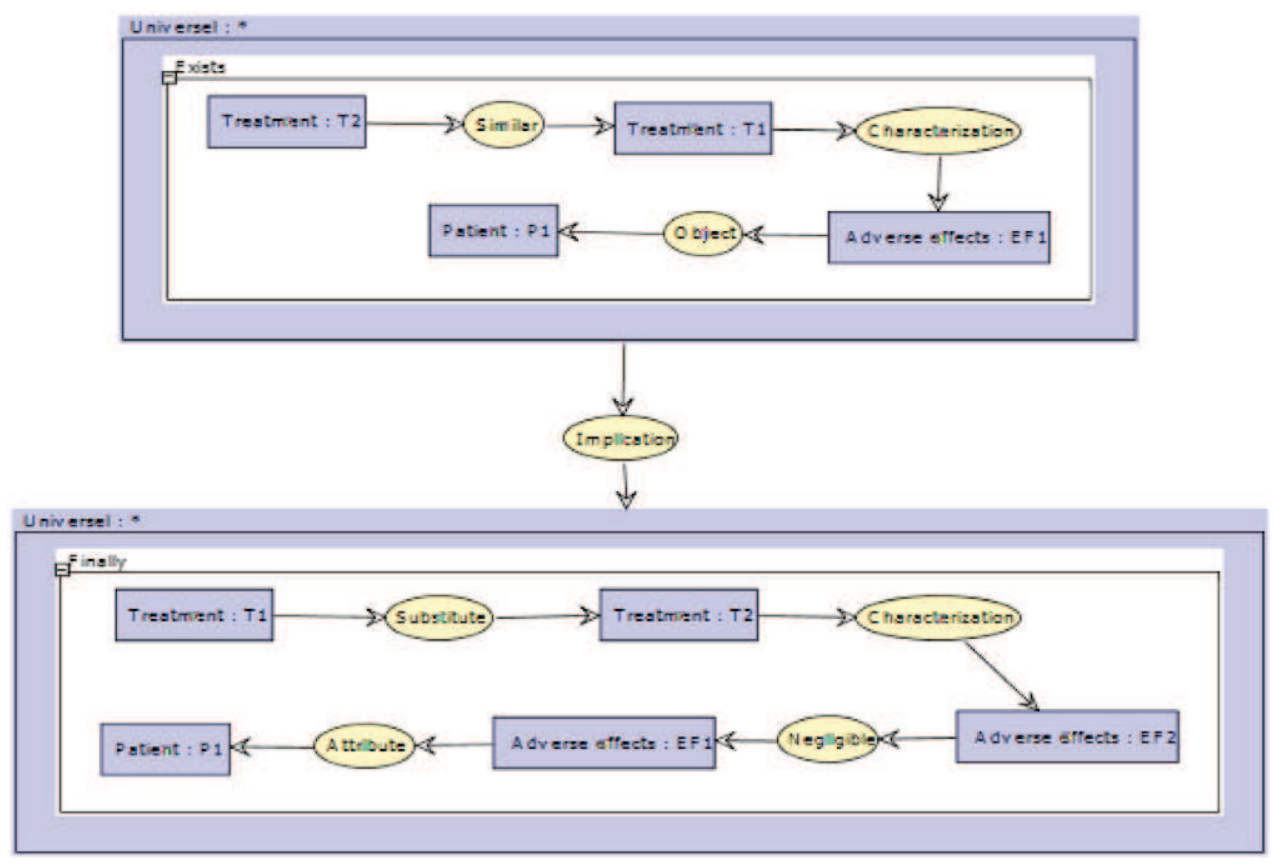

Fig. 3. Third property. 
Table 3

Hierarchy of concepts

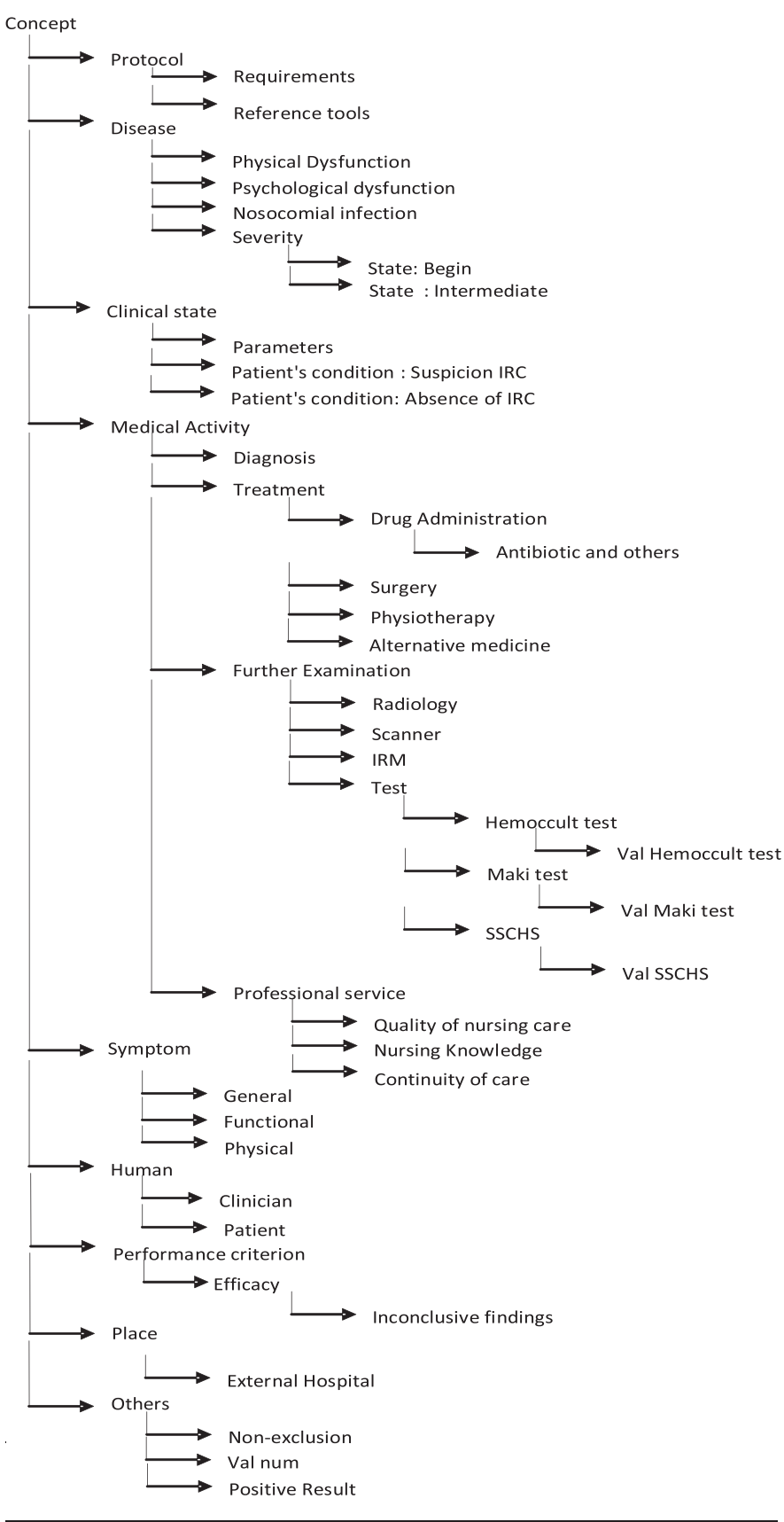

The verification procedures are fully descriptive graphs because all knowledge is represented by graphs, and all reasoning mechanisms are used by graph operations [9]. This allows for the visualization of the reasoning and the use of the structural properties of the graphs to verify compliance with the CRI protocol-specified requirements (good medical practice, hospital characteristics, protocol goal, and patient clinical condition). During the verification process, the protocol is proven to not contain CRI abnormalities intrinsic to its definition and to comply with the various types of temporal properties useful for its effective implementation.

In Fig. 4, we see a part of the medical protocol for the detection of an CRI.

First, the patient is in a state where he/she is not suspected of having an CRI.
If the patient has specific symptoms, such as signs of a local infection or a high fever, the relation "symptomsAppear" triggers the process of examining for a case of an CRI.

Three tests are performed in parallel (Maki Test (a semi-quantitative culture method rolling the catheter segment across blood agar), Hemoccult Test (a qualitative assay for occult blood in stool based on detecting the peroxidase activity of hemoglobin), Study of Semiquantitative Cultures of Catheter Hub and Skin) to verify the patient's condition and whether he/she is suspected of contracting an CRI.

We then translate this model into a conceptual graph formalism, thus introducing the notion of temporality in this formalism for temporal context representation.

"If the numerical value of the MakiTest is equal to 3 and the numerical value of the Hemoccult Test is equal to 2, then the patient is not suspected of contracting an CRI."

Table 4

Hierarchy of relationships

Relation

Usua

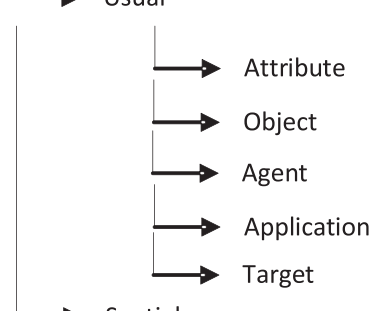

Spatial

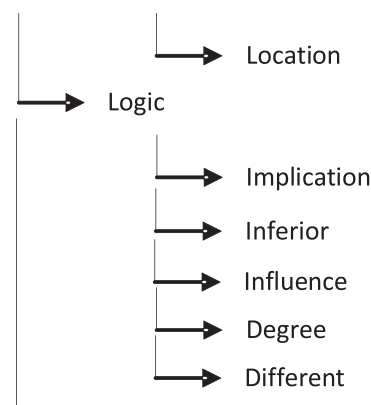

empora
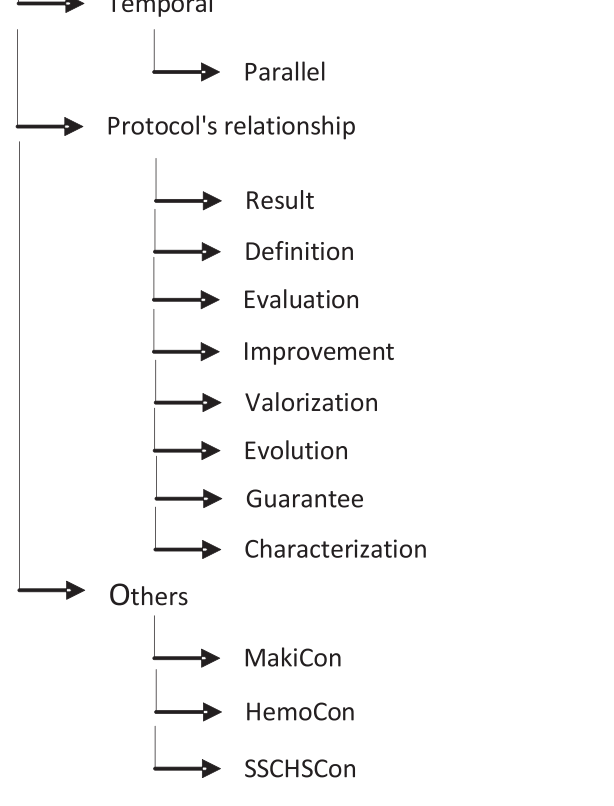


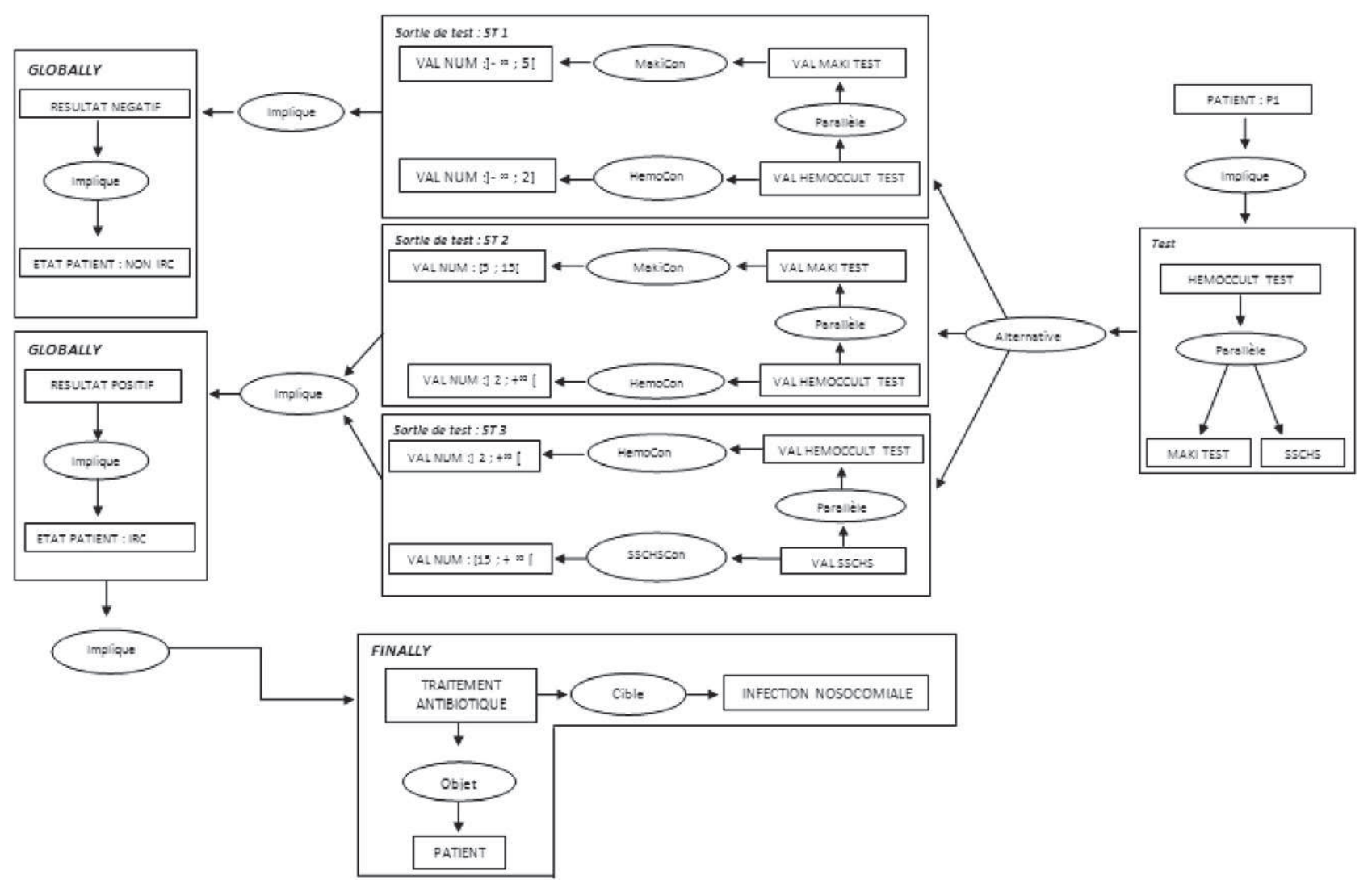

Fig. 4. Situational graph of an CRI or IRC.

\subsubsection{Query graph}

"If the numerical value of $\quad \square \neg$ (stateTop_R==PatientIsntSuspectedToHaveIRC) the MakiTest is equal to 3 and the numerical value of the Hemoccult Test is equal to 2, then the patient is not suspected of contracting a nosocomial disease" (Fig. 5).

The projection is the fundamental operation of reasoning in the formalism of conceptual graphs. This projection is a type of graph matching, which can be easily visualized and interpreted. The projection mechanism performs a computation specialization between the graphs that takes into account the links described in the specialization hierarchies of concepts, relationships, and temporal modalities.

For example, if we want to answer the question "Does the property represented by graph (query) $\mathrm{H}$ present a target situation also described by graph G?" This search operator information allows us to respond and to verify the property, and it will seek the possible projections of $\mathrm{H}$ in $\mathrm{G}$.

We apply this principle by translating the graph's first situation with the above model and then compare it to the query graph and decide whether to validate the model.

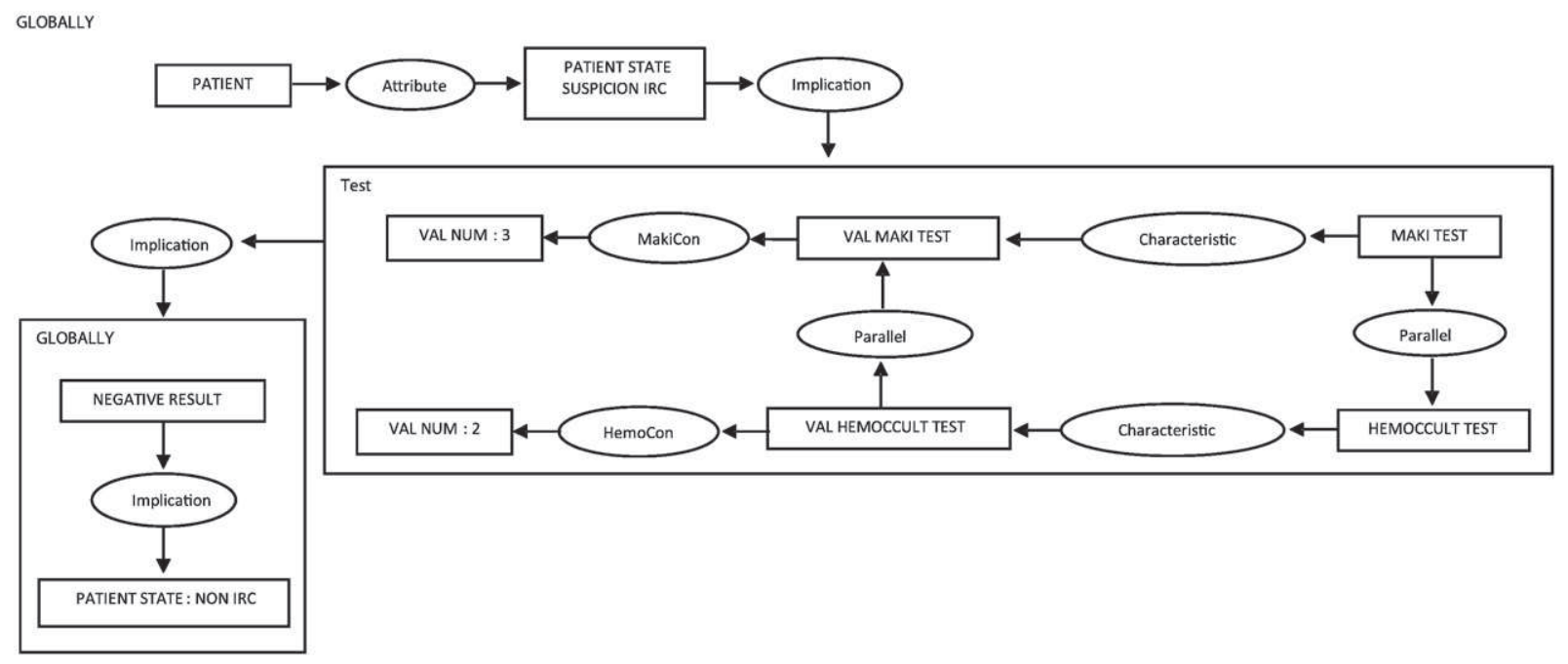

Fig. 5. Query graph of the CRI or IRC. 
In fact, we made 2 types of translations from the CRI model into conceptual graphs:

\subsubsection{Translation of the situational graph}

Several test outputs are derived from this model, which involve appropriate antibiotic treatments, or not, according to the patient's condition.

Indeed, a patient is suspected of contracting an CRI if:

- The value of the Maki test is between 5 (inclusive) and 15 (excluded), and the value of the Hemoccult test is between 2 (excluded) and $+\infty$.

- The value of the Hemoccult test is between 2 (excluded) and $+\infty$, and the value of the SSCHS test is between 15 (inclusive) and $+\infty$.

The patient is not suspected in the case where:

- The value of the Maki test is between $-\infty$ and 5 (excluded), and the value of the Hemoccult test is between $-\infty$ and 2 (inclusive).

\subsubsection{Translation of the query graph}

Setting a query in the form of a graph question, we cannot prove the suspicion of a patient contracting a nosocomial disease. This evidence projection query graph in the situational graph is based on a calculation of a specialization between the 2 graphs.

The query graph with a numerical value equal to 3 in the MakiTest and a numerical value equal to Hemoccult Test 2 projects into the test output ST1 situational graph.

In fact, we have the 2 following membership relations:

- $3 \in]-\infty ; 5[$

$-2 \in]-\infty ; 2]$
From the visual modeling and temporal reasoning, graph operations are arranged sequentially. We then translate the four basic requirements of medical protocols (good medical practice, hospital characteristics, purpose of the protocol, clinical condition of the patient) in the form of graphs to specify properties and structure the information to make reasoning that orient choices and decisions. This verification includes a description (planned results and delivery strategy) and a graphical illustration (a logic model), which demonstrate how the activities of checking are expected to lead to the achievement of the planned results.

From the graphical representation provided by Fig. 6, we can deduce that the graph satisfies the conditions of the query graph property, thereby validating the model. Our approach has explored the potential of visual reasoning as a suitable tool in solving problems related to semantic knowledge formalization and verification.

\section{Discussion}

Establishing effective strategies for problem solving requires imagination and ingenuity, which is also true for the formulation of the problem in the implementation of its resolution. Designing relevant specifications and an interesting model for the problem generally provide a better overview, which will provide analysts valuable elements to take full advantage of the use of visual reasoning. Emphasis should be placed on techniques that provide analysts with valuable information to facilitate a general understanding of the problems and to determine the best way to influence the efficiency of the resolution process.

From the point-of-view of knowledge engineering, diagrams have the advantage of incorporating reasoning mechanisms, which are more easily engaged for the encoding and handling of structures and

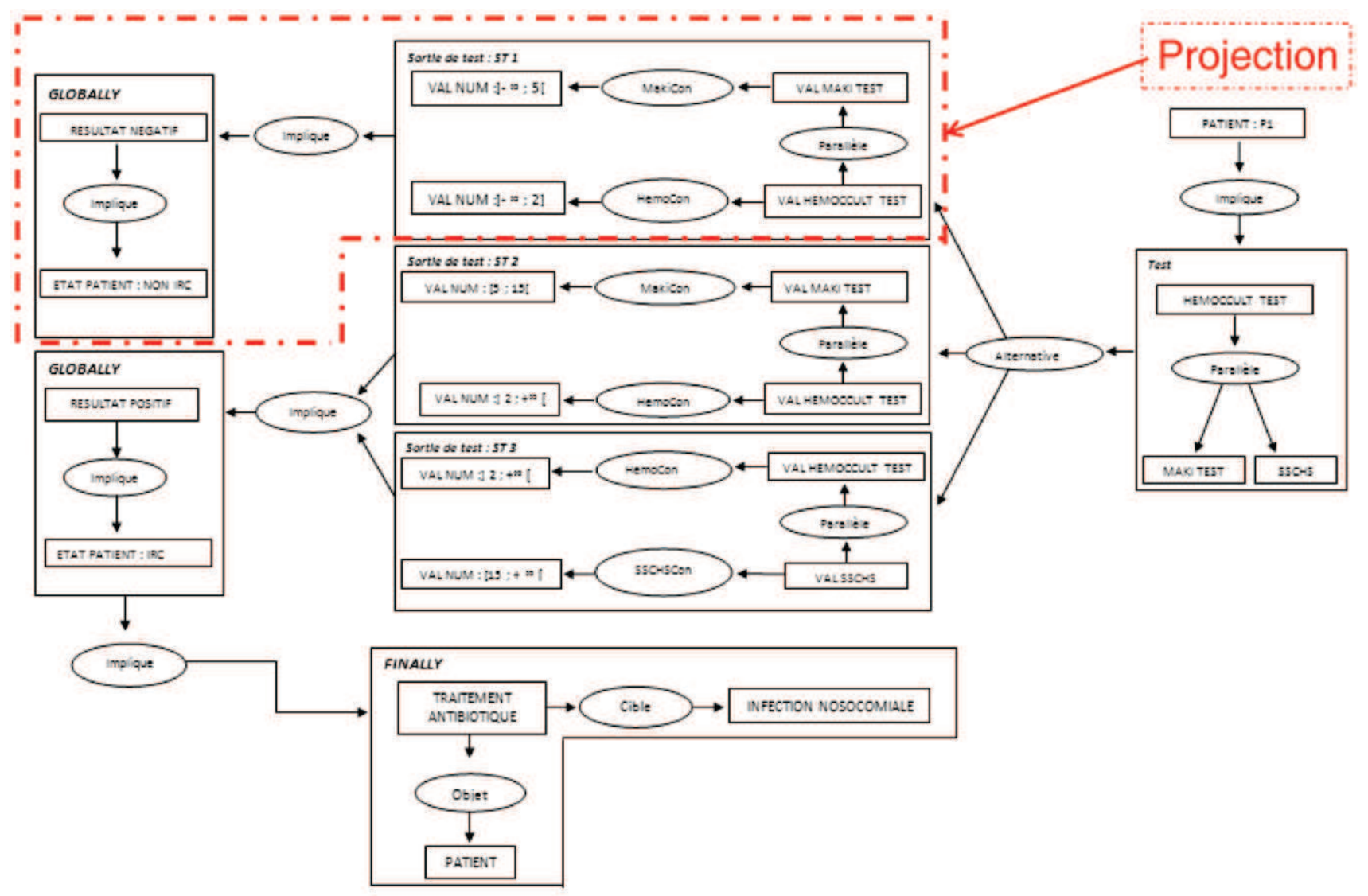

Fig. 6. Intravenous catheter-related infection (CRI or IRC) result of the projection of the query graph in the situational graph. 
strategies for solving problems [22]. Therefore, it follows that charts show a potential efficiency higher than that of first-order logic. In this case, it is not necessary to go through an entire database to find everything about the target concept [23].

In our case, the model is a conceptual graph, and through its manipulation, it can generate valuable insights into the conceptualization of the problem. The cognitive values of this model are its ability to generate views from conceptual graphs for the visualization of the knowledge that they contain. The automatic generation of this visualization is important for both the validation of graphs and the communication of the knowledge that they convey. In terms of logic, this process promotes a technical demonstration, insofar as the perception of some evidence that naturally flows, from a graphical representation, may be more difficult to establish from an axiomatic description.

Finally, the visual illustration of the steps of the reasoning steps is likely to promote the implementation of creative methods of problem solving or at least stimulate the development of alternative ways of problem solving, which demonstrates that typical proofs include visualized tasks, complementary solutions, and justifications for the performed actions.

At the semantic level, we opt for a formal structure to interpret each symbol as a primitive form acceptable to some grammar and to subsequently facilitate a recognition process more complex than syntactic construction. In this context, the main formal grammar is that of CTL, which provides a rigorous framework for describing knowledge from a process of feedback. This formalization of model representations and the reasoning mechanisms involved may provide the means to guide model capitalization and the exploitation of knowledge.

\section{Conclusion}

The formalization of knowledge is valuable in the field of complex nosocomial pathologies. This explicit knowledge is very useful because official figures show that in France 6\% to 7\% of hospitalizations are complicated by a nosocomial infection that is often serious. This number varies depending on the service in which the person is hospitalized but can indeed reach $30 \%$ in a service such as Resuscitation. Nosocomial diseases also have a noticeable economic burden.

The development of information technology and communication in the professional world has been swift and massive. One of the effects of new technologies on information and communications is the increasing need to present information visually [24]. However, this raises some interesting questions: what is the logical status of reasoning that uses visualization? What are the cognitive advantages and pitfalls of this reasoning?

The implementation of an audit approach with logical foundations is valuable; however, with graphic reasoning paths, it is intuitive to facilitate the understanding of the verification steps by the actors of a domain [25]. The visual transformation rules that are applied are only valid if, and only if, their demonstration can be proven using only visual rules and if mistakes cannot be derived from this evidence schematic. Thus, inference rules are equally powerful graphically with valid explanations and more interactive than their textual counterparts [26]. Therefore, diagrams can be used as a valid basis for formal proofs, defining a system of adequate and consistent reasoning vis-àvis formal logic. More generally, tools with diagram evidence are used to support the development of schematic reasoning by providing visual representations of formal proofs that shall draw attention to the appropriate warning situations [27]. The progress of our approach better highlights the causes of noncompliance with certain properties or at least suggests some improvements in failures of evidence.

We can also add that our research is not only applicable to the medical field but can also be applied in many other sectors [28-31]. In fact, in an industrial setting, for example, our methods can be applied to check quality protocols, whereas in scientific research, they can be applied to experimental protocols involving the description of requirements and to conduct an experiment or test.

\section{References}

[1] Samuel SO, Kayode OO, Musa OI, Nwigwe GC, Aboderin AO, Salami TAT, et al, Nosocomial infections and the challenges of control in developing countries. Afr J Clin Exp Microbiol 2010;11(2):102-10.

[2] Benenson AS, editor. Control of communicable diseases manual. 16th ed. Washington (USA): The. American Public Health Association; 1995.

[3] Horan TC, Gaynes RP. Surveillance of nosocomial infections. In: Mayhall CG, editor. Hospital Epidemiology and Infection Control. 3rd ed. Philadelphia: Lippincott Williams \& Wilkins; 2004. p. 1659-702.

[4] $\mathrm{WHO} / \mathrm{CDS} / \mathrm{CSR} / \mathrm{EPH}$. Prevention of hospital-acquired infections. A practical guide2nd ed. . World Health Organization Department of Communicable Disease, Surveillance and Response; 122002.

[5] Pérez B, Porres I. Authoring and verification of clinical guidelines: a model driven approach. J Biomed Inform 2010;43:520-36.

[6] Scheckler WE, Brimhall D, Buck AS, Farr BM, Friedman C, Garibaldi RA, et al. Requirements for infrastructure and essential activities of infection control and epidemiology in hospitals: a consensus panel report. Am J Infect Control February 1998;26(1):47-60.

[7] Grumberg O, Veith H. 25 years of model checking: history, achievements, perspectives. , VIISpringer978-3-540-69849-4; 2008 [231 pages].

[8] Sowa JF. Conceptual structures: information processing in mind and machine. The Systems Programming Series (Hardcover). Boston, Massachusetts, USA: AddisonWesley Longman Publishing Co., Inc.; 1984481.

[9] Baget J-F, Mugnier M-L. Extensions of simple conceptual graphs: the complexity of rules and constraints. J Artif Intell Res 2002;16:425-65.

[10] Sowa JF. Knowledge representation: logical, philosophical, and computational foundations. Pacific Grove, California (U.S.A): Brooks Cole Publishing Co0-53494965-7; 2000 [608 pages].

[11] Chein M, Mugnier M-L. Graph-based knowledge representation: computational foundations of conceptual graphs. Series: Advanced Information and Knowledge Processing. London (United Kingdom): Springer978-1-84800-285-2; October 2008 [445 pp.].

[12] Baader F, Calvanese D, McGuinness DL, Nardi D, Patel-Schneider PF. The description logic handbook: theory, implementation, applications. Cambridge, UK: Cambridge University Press0-521-78176-0; 2003.

[13] Yao H, Etzkorn L. Automated conversion between different knowledge representation formats. Knowl-Based Syst 2006;19(6):404-12.

[14] Argüello Casteleiro M, Des Diz JJ. Clinical practice guidelines: a case study of combining OWL-S, OWL, and SWRL. Knowl-Based Syst April 2008;21 (3):247-55.

[15] Horrocks I, Patel-Schneider PF, Bechhofer S, Tsarkov D. OWL rules: a proposal and prototype implementation. Web Semantics: Science Services and Agents on the, World Wide Web, Vol. 3, Issue 1; July 2005. p. 23-40.

[16] Shadbolt N, Hall W, Berners-Lee T. The semantic web revisited. IEEE Intell Syst 2006;21(3):96-101.

[17] Dau F, Eklund P. A diagrammatic reasoning system for the description logic. J Vis Lang Comput 2008;19:539-73.

[18] Clarke EM, Emerson EA, Sistla AP. Automatic verification of finite-state concurrent systems using temporal logic specifications. ACM Trans Program Lang Syst 1986;8 (2):244-63. http://dx.doi.org/10.1145/5397.5399.

[19] Moulin B. Temporal contexts for discourse representation: an extension of the conceptual graph approach. Appl Intell 1997;7:227-55.

[20] López-Cuadrado JL, Colomo-Palacios R, González-Carrasco I, García-Crespo A, Ruiz-Mezcua B. SABUMO: towards a collaborative and semantic framework for knowledge sharing. Expert Syst Appl August 2012;39(10):8671-80.

[21] Musen MA. The knowledge acquisition workshops: a remarkable convergence of ideas. Int J Hum Comput Stud February 2013;71(2):195-9.

[22] Luger GF. Artificial intelligence: structures and strategies for complex problem solving. 6th ed. Addison Wesley; March 72008 [Hardcover: 784 pages].

[23] Russell S, Norvig P. Artificial intelligence: a modern approachIn: Prentice Hall, editor. Third edition. ; Décembre 12009 [1152 pages].

[24] Allwein G, Barwise J. Logical reasoning with diagrams (studies in logic and computation). First ed. OUP USA; July 181996.

[25] Kamsu-Foguem B, Tchuenté-Foguem G, Foguem C. Using conceptual graphs for clinical guidelines representation and knowledge visualization. Information Systems Frontiers. http://dx.doi.org/10.1007/s10796-012-9360-2.

[26] Kamsu-Foguem B, Diallo G, Foguem C. Conceptual graph-based knowledge representation for supporting reasoning in African traditional medicine. Engineering Applications of Artificial Intelligence April 2013;26(4):1348-65.

[27] Kamsu-Foguem B, Tchuenté-Foguem G, Allart L, Zennir Y, Vilhelm Y, Mehdaoui H, Zitouni D, Hubert H, Lemdani M, Ravaux P. User-centered visual analysis using a hybrid reasoning architecture for intensive care units. Decision Support Systems December 2012;54(1):496-509.

[28] Kamsu-Foguem B. Knowledge-based support in Non-Destructive Testing for health monitoring of aircraft structures. Advanced Engineering Informatics October 2012;26(4):859-69.

[29] Kamsu-Foguem B, Rigal F, Mauge F. Mining association rules for the quality improvement of the production process. Expert Systems with Applications March 2013;40(4):1034-45.

[30] Kamsu-Foguem B, Mathieu Y. Software architecture knowledge for intelligent light maintenance. dvances in Engineering Software January 2014;67:125-35.

[31] Kamsu-Foguem B, Flammang A. Knowledge description for the suitability requirements of different geographical regions for growing wine. Land Use Policy 2014;38C:719-31. 